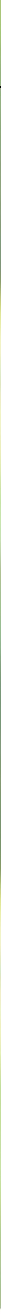

$k a$ und der technische Fortschritt erlauben eine frühe ambulante Krebstherapie. Für Ärzte und Patienten oft eine Herausforderung.

Der Patient ist mehr denn je gefragt

\title{
Orale Krebstherapien erfordern eine aktive Mitarbeit
}

Schwerpunkt der klinischen onkologischen Forschung ist die Entwicklung neuer und immer wirksamerer, kurativer Interventionen. Jedoch ist mit dem Nachweis eines signifikanten Erfolgs einer Arzneimittelwirkung nicht automatisch dessen Nutzen gesichert. Dieser ist von vielen Faktoren abhängig, die oft erst in der Praxis relevant werden. Entscheidend sind hier unter anderem das Alter des Patienten, das Ausmaß von Komorbiditäten verbunden mit altersrelevanter Polypharmazie und abnehmender Bioverfügbarkeit durch reduzierte Resorption im Gastrointestinaltrakt. Hinzu kommen Arzneimittelinteraktionen, der Performancestatus, die Lebens- und Essensgewohnheiten des Patienten usw. Vielfältige Einflüsse also, die sich nachhaltig auf die Wirkung der Therapie - bei jedem einzelnen Betroffenen unterschiedlich - auswirken und damit den Nutzen der Behandlung modulieren. Hinzu kommen - in anderen Ländern und zunehmend auch bei uns - die materiellen und immateriellen Kosten im Zusammenhang mit der Krebsbehandlung.

\section{Adhärenz, ein immer noch unterschätzter Faktor}

Immer noch zu wenig Beachtung findet in diesem Zusammenhang die Adhärenz des Kranken. Dabei ist sie von kritischer Be-

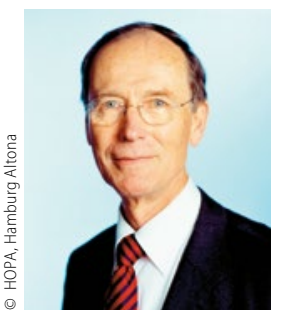

Prof. Dr. Ulrich R. Kleeberg

Hämatologisch-onkologische

Praxis Altona, Tagesklinik Struensee-Haus,

Hamburg

urkleeberg@hopa-hamburg.de

deutung für den Behandlungserfolg. Wie wichtig die Mitarbeit des Patienten an seiner Genesung ist, zeigen verschiedene Erkenntnisse, die erst in jüngerer Zeit wissenschaftlich bestätigt wurden: Rauchen etwa mindert den Effekt einer Chemo- und/ oder Strahlentherapie, die Aufnahme verschiedener Wirkstoffe ist abhängig von der resorptiven Kapazität des (oberen) Gastrointestinaltrakts. Diese wiederum wird durch eine Reihe von Faktoren bestimmt: von der exokrinen und Schleimhautfunktion, von Nahrungsmittelinteraktionen, z. B. bei gleichzeitiger 


\section{Hier steht eine Anzeige.}

Springer 
Aufnahme von fetthaltiger Nahrung oder auch Milch, von der Verweildauer im Verdauungstrakt, von Alkoholkonsum als relevantem Wachstumsfaktor für Tumorzellen und nicht zuletzt vom Vorliegen entzündlicher, die Tumorprogredienz fördernder Veränderungen usw.

\section{Gemeinsam an einem Strang ziehen}

Gerade im Hinblick auf die Adhärenz des Patienten darf sich die Aufgabe des Arztes also nicht nur auf die Rezeptur beschränken, etwa auf das „Therapieschema“, welches vielleicht auch noch an die Arzthelferin delegiert wird. Vielmehr bedarf es, um eine optimale Bioverfügbarkeit des Pharmakons sicherzustellen, einer konzertierten Aktion des onkologischen Teams und wo immer möglich der Mitarbeit des Patienten selbst und seiner Angehörigen.

Vor dem Hintergrund dieser Vielzahl von Interaktionen und Wechselwirkungen, also der variablen Bioverfügbarkeit der Medikationen, die Adhärenz des Patienten nicht ernst zu nehmen, ist fahrlässig. Die Kosten sind hoch:

Für die Kranken sind es die Einbußen an Überlebenschancen, dies konnten Jacquie H. Chirgwin und Kollegen im Rahmen der BIG 1-98-Studie wieder eindrucksvoll zeigen [Chirgwin JH et al. J Clin Oncol. 2016;34(21):2452-9; siehe dazu auch den Journal-Club-Beitrag Seite 24], und das mit einem weiteren Tumorprogress verbundene Leid.

Für die Gemeinschaft sind es die erhöhten Kosten für palliative Maßnahmen. Diese können bei den ausufernden Preisen für die bereits eingeführten und noch bevorstehenden Innovationen, beispielsweise die verschiedenen Tyrosinkinasehemmern oder die Immuncheckpointinhibitoren, zu einer relevanten Belastung des Gesundheitswesens werden.

\section{Handlungsbedarf in drei Feldern}

Kurzum, die Ärzteschaft, aber ganz besonders die Onkologie, muss sich dieses Problems einer verminderten Arzneimittelwirkung infolge mangelnder Adhärenz aktiv zuwenden und Strukturen schaffen, die die Wirkung eines Pharmakons auch in Nutzen für den Patienten übersetzen. Drei Aufgabenbereiche stehen oben an, der Allgemeinzustand des Patienten, die Toxizität der Therapie und deren effektive Behandlung:

- Bestehen ein reduzierter Allgemeinzustand, eine negative bzw. depressive Grundstimmung, Komorbidität verbunden mit Polypharmazie, höheres biologisches Alter, Vergesslichkeit, alleinstehender Sozialstatus, dann müssen Strategien eröffnet werden, mit denen man die Adhärenz nachhaltig ver- bessern kann: Beispiele dafür können eine wöchentliche Vorausplanung der täglichen Tabletteneinnahme (in entsprechenden Döschen erhältlich über die Apotheken) sein, die Verblisterung der Tagesdosis und deren Zustellung begrenzt auf wenige Tage, oder die Überprüfung der Compliance gemeinsam mit dem Apothekenpersonal, den Mitarbeitern, der Krankenpflege; alles begleitet von einer engagierten und regelmäßigen psychosozialen Anteilnahme zur Motivation des Patienten.

- Insbesondere bei der Nachsorge von Patienten mit kurativ intendierter Primärtherapie muss der Adhärenz bei adjuvanten Maßnahmen besonderes Augenmerk gewidmet werden. Wo immer es möglich ist, sollten dabei die Angehörigen mit einbezogen werden. Studien zu Disease-Management-Programmen haben den Wert eines solchen Vorgehens als hilfreich belegt [Jacob L et al. Breast Cancer Res Treat. 2015;153(2):391-5]

- Von besonderer Bedeutung ist schließlich die Kontrolle - im Sinne von Nachfragen, Vorbeugen und Behandeln - spezifischer Nebenwirkungen. So hat sich bei Brustkrebspatientinnen mit adjuvanter Hormonersatztherapie, also kontinuierlicher endokriner Therapie, gezeigt, dass es hilft, wenn sie aktiv den möglichen Nebenwirkungen entgegentreten können, beispielsweise durch tägliche Bewegung und Krankengymnastik [Irwin ML et al. J Clin Oncol. 2015;33(10):1104-11], durch Vitamin-D-Substitution bei Arthralgien mit niedrigen Serumwerten [Khan QJ et al. Breast Cancer Res Treat. 2010; 119(1):111-8] oder durch Wechselduschen bei vasomotorischen Beschwerden. Auch eine zeitlich begrenzte zusätzliche medikamentöse Therapie, die die Komplikationen der endokrinen Therapie lindert (nicht-steroidale Antirheumatika bei Arthralgien, Gabapentin bei Hitzewallungen etc.), kann erwogen werden.

Zusammen genommen ist dies der Versuch, die Lebensführung der Betroffenen nachhaltig zu beeinflussen. Letztlich geht es darum, ein Bewusstsein dafür zu schaffen, dass eine mangelnde Adhärenz die Bioverfügbarkeit der Krebstherapie so stark herabsetzt, dass ihre Wirkung und der Nutzen insgesamt bezweifelt werden müssen. Gemeinsam mit dem Patienten, mit seinen Angehörigen und dem onkologischen Team muss es unser Ziel sein, dem entgegenzuwirken. In diesem Bemühen sind alle an der Betreuung des Krebspatienten Beteiligten gefordert, auch der Ökotrophologe oder der beratende Apotheker.

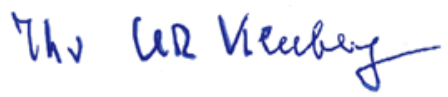

